\title{
The long non-coding RNA LNC_000397 negatively regulates PRRSV replication through induction of interferon-stimulated genes
}

Jing Zhang ${ }^{*}$, Lipeng Gan, Pu Sun, Jian Wang, Dong Li, Yimei Cao, Yuanfang Fu, Pinghua Li, Xingwen Bai, Kun Li, Xueqing Ma, Huifang Bao, Yingli Chen, Jie Zhang, Zaixin Liu* and Zengjun Lu*

\begin{abstract}
Background: Porcine reproductive and respiratory syndrome virus (PRRSV) is one of the most significant threats to the global swine industry. It is of great importance to understand viral-host interactions to develop novel antiviral strategies. Long non-coding RNAs (IncRNAs) have emerged as critical factors regulating host antiviral immune responses. However, IncRNAs participating in virus-host interactions during PRRSV infection remain largely unexplored.

Method: RNA transcripts of porcine alveolar macrophages (PAMs) infected with two different PRRSV strains, GSWW/2015 and VR2332, at $24 \mathrm{~h}$ post-infection were sequenced by high-throughput sequencing. Four programs namely, CNCl, CPC, PFAM, and phyloCSF, were utilized to predict the coding potential of transcripts. mRNAs colocalized or co-expressed with differentially expressed IncRNAs were considered as their targets. Fuction of IncRNAs was predicted by GO and KEGG analysis of their target mRNAs. The effect of LNC_000397 on PRRSV replication was validated by knockdown its expression using siRNA. Target genes of LNC_000397 were identified by RNA-Sequencing and validated by RT-qPCR.
\end{abstract}

Result: In this study, we analyzed IncRNA and mRNA expression profiles of PRRSV GSWW/2015 and VR2332 infected porcine alveolar macrophages. A total of 1,147 novel IncRNAs were characterized, and 293 IncRNAs were differentially expressed. mRNAs co-localized and co-expressed with IncRNAs were enriched in pathogen-infection-related biological processes such as Influenza A and Herpes simplex infection. Functional analysis revealed the IncRNA, LNC_000397, which was up-regulated by PRRSV infection, negatively regulated PRRSV replication. Knockdown of LNC_000397 significantly impaired expression of antiviral ISGs such as MX dynamin-like GTPase 1 (MX1), ISG15 Ubiquitin-like modifier (ISG15), and radical S-adenosyl methionine domain containing 2 (RSAD2).

Conclusions: LNC_000397 negatively regulated PRRSV replication by inducing interferon-stimulated genes (ISGs) expression. Our study is the first report unveiling the role of host IncRNA in regulating PRRSV replication, which might be beneficial for the development of novel antiviral therapeutics.

\footnotetext{
*Correspondence: zhangjing@caas.cn; liuzaixin@caas.cn; luzengjun@caas.cn

State Key Laboratory of Veterinary Etiological Biology, OIE/National

Foot-and-Mouth Disease Reference Laboratory of China, Lanzhou Veterinary Research Institute, Chinese Academy of Agricultural Sciences,

Xujiaping No.1, Yanchangpu, Lanzhou 730046, Gansu, China
} permits use, sharing, adaptation, distribution and reproduction in any medium or format, as long as you give appropriate credit to the original author(s) and the source, provide a link to the Creative Commons licence, and indicate if changes were made. The images or other third party material in this article are included in the article's Creative Commons licence, unless indicated otherwise in a credit line to the material. If material is not included in the article's Creative Commons licence and your intended use is not permitted by statutory regulation or exceeds the permitted use, you will need to obtain permission directly from the copyright holder. To view a copy of this licence, visit http://creativecommons.org/licenses/by/4.0/. The Creative Commons Public Domain Dedication waiver (http://creativeco mmons.org/publicdomain/zero/1.0/) applies to the data made available in this article, unless otherwise stated in a credit line to the data. 
Keywords: PRRSV, IncRNA, RNA-Sequencing, Interferon, Antiviral

\section{Introduction}

Porcine reproductive and respiratory syndrome (PRRS) is one of the most severe swine diseases worldwide. It is characterized by reproductive failures in sows and respiratory illness in growing pigs, which has caused an enormous economic burden on the swine industry globally. The etiological agent, Porcine reproductive and respiratory syndrome virus (PRRSV), is an enveloped, positive single-stranded RNA virus that belongs to the family Arteriviridae of the order Nidovirales [21]. The genome size of PRRSV varies from 14.9 to $15.5 \mathrm{~kb}$ in length, with 11 open reading frames (ORFs). ORF1a and ORF1b, which occupy about $70 \%$ of the viral genome, encode two long polyproteins, pp1a and pplab. Subsequently, proteolytic processing of these polyproteins yields at least 14 non-structural proteins (nsps), which include four proteases (NSP1 $\alpha$, NSP1 $\beta$, NSP2, and NSP4), the RNA-dependent RNA polymerase (NSP9), a helicase (NSP10), and an endonuclease (NSP11). ORFs 2-7 encode structure proteins including, glycosylated membrane proteins GP2-GP5, a non-glycosylated membrane protein $(\mathrm{M})$, and the nucleocapsid (N) protein. [5].

The virus was initially isolated in Europe and the United States in the early 1990s and categorized into the European PRRSV and the North American PRRSV genotypes [28]. In 2006, highly pathogenic PRRSV (HPPRRSV), a novel variant of type 2 PRRSV featured by the 30-aa deletion in the coding region of non-structure protein 2, emerged in China [12]. The clinical features of HP-PRRSV infections are high fever, high morbidity, and fatality. Since its first outbreak, HPPRRSV has caused an inestimable economic loss. A novel NADC30-like virus with 133-aa deletion in the nsp2 was isolated in the field in 2013 [36]. It has been reported that NADC30-like viruses are undergoing recombination with HP-PRRSV, which makes disease control more challenging.

PRRSV infection impairs host innate and adaptive immune responses, leading to ineffective virus clearance and the establishment of chronic infection. The type I interferon response is a critical component of the host innate immune response against virus infection. The receptor for type I IFNs is ubiquitously expressed. Upon IFN binding, transmits the signal through two receptor-associated tyrosine kinases, tyrosine kinase 2 (TYK2) and Janus kinase 1 (JAK1), resulting in the phosphorylation of signal transducers and activators of transcription STAT1 and STAT2. The phosphorylated
STATs form heterodimers and bind to IFN-regulatory factor 9 (IRF9) to form the active transcription factor complex IFN-stimulated gene factor 3 (ISGF3). ISGF3 initiates transcription by binding to the promoters of interferon-stimulated genes (ISGs), which contain IFNstimulated response elements (ISREs) [26, 27]. Some ISGs encode proteins with direct antiviral activity, such as ISG15, Mx1, Viperin, and interferon-inducible RNAdependent protein kinase(PKR). Many of the ISGs encode transcription factors that enhance the production of interferons and other cytokines.

PRRSV has developed a set of mechanisms for suppressing interferon signaling. Several viral proteins have been reported to impair IFN production (Nsp1, Nsp2, Nsp4, Nsp11, and N protein) [22]. Nsp1 translocates to the nucleus, inhibits the binding of transcription factor IRF3 with CBP, and facilities CBP degradation, resulting in down-regulation of IFN promoter activity [8]. Nsp1 $\beta$ restrains STAT1 phosphorylation and ISGF3 nuclear translocation. Nsp2 inhibits $\mathrm{NF}-\mathrm{KB}$ activation by interfering with the polyubiquit-

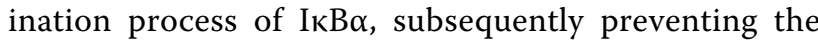
degradation of the ІкB $\alpha$ protein, which are required for the releasing of NF- $\mathrm{kB}$ dimers and their translocation to the nucleus, where they regulate transcription of type I interferons [23]. Nsp11 induces STAT2 degradation, and it also interacts with IRF9 to impair the nuclear translocation of the ISGF3 complex [33]. Nsp11 and $\mathrm{N}$ protein both inhibits IRF3 phosphorylation and nuclear translocation to prevent IFN transcription [19]. $\mathrm{N}$ protein also interacts with SOCS1 (a negative regulator of JAK-STAT signaling) to inhibit IFN production [14].

Non-coding RNAs include long ncRNAs (lncRNAs) and short ncRNAs such as microRNAs (miRNAs), PIWI-interacting RNAs (piRNAs), and small nuclear RNAs (snRNAs) [6]. By definition, lncRNAs are transcripts lacking protein-coding potential and longer than $200 \mathrm{nt}$. The majority of lncRNAs are 7-methylguanosine-capped, spliced, and polyadenylated. A number of reports have delineated the critical roles of IncRNAs in a wide variety of biological processes [15]. Precise regulation of gene expression in the immune system is crucial to protect hosts from pathogen infections by generating effective immune responses while limiting autoimmunity [20]. Emerging evidence has established that lncRNAs are involved in the regulation of immune cell differentiation and immune responses. LncRNAs modify the virus-host interactions by regulating ISG 
expression or through ISG independent manner, such as stabilizing virus RNA structure or modulating viral replication [17, 18, 25-27].

The role of host proteins and microRNAs in virushost interactions of PRRSV has been extensively studied. However, the function of host lncRNAs in regulating PRRSV-induced immune response is largely unknown. Transcriptomic profiles of PRRSV infected host cells have been analyzed either in vitro or in vivo [1, 29, 31]. These studies revealed that PRRSV infection resulted in alteration of gene expression evolved in innate immune response signaling pathways such as type I IFN signaling, TLR, and RIG-I signaling. MicroRNAs also play critical roles in regulating host innate immunity. For example, miR-24-3p facilitates PRRSV replication by suppression of HO-1 expression [31]. MiR-373 promotes PRRSV replication through impairing type I IFN production [4]. However, the functions of lncRNAs in host immune response against PRRSV are unknown. In our previous study, we analyzed expression profiles of HP-PRRSV strain GSWW/2015 and FL-12 infected PAMs by RNAsequencing to identify lncRNAs regulated by PRRSV infection. We found a lncRNA, TCONS_00054158, induced by different strains of PRRSV and poly (I: C) but not by the heat-inactivated virus. The function of this lncRNA is under study in our lab (Zhang et al., 2017b). Zhen and colleagues analyzed mRNA, IncRNA, and microRNA profiles of PRRSV infected PAMs from two pig breeds [35]. Another study used co-expression network analysis of differently expressed mRNAs and lncRNAs after PRRSV infection to identify the function of these lncRNAs. They found some of the lncRNAs associated with the interferon-induced genes [30].

To explore the role of lncRNAs in the virus-host interaction of PRRSV, we sequenced RNA transcripts of PAMs infected with GSWW/2015 (GSWW for short) and vaccine strain VR2332 at $24 \mathrm{~h}$ post-infection. Using rigorous methods to evaluate the coding potential of the transcripts, 1,147 novel lncRNAs were characterized, and a total of 293 lncRNAs were differentially expressed. We identified a lncRNA, LNC_000397, which was upregulated after PRRSV infection and negatively regulated PRRSV replication by inducing ISGs. This research firstly reported the function of IncRNA in regulating PRRSV replication, which might be beneficial for understanding the interaction between PRRSV and the host immune system.

\section{Material and methods}

\section{Cells and viruses}

PAMs were isolated from the lung lavage fluid of 4-weekold SPF (Specific Pathogen-Free) pigs as described previously [34]. PAMs were maintained in RPMI-1640 medium, and Marc-145 cells were cultured in DMEM. Both culture media were supplemented with $10 \%$ heatinactivated FBS (GIBCO), $100 \mathrm{U} / \mathrm{ml}$ penicillin, and $100 \mathrm{mg} / \mathrm{ml}$ streptomycin. Cells were incubated at $37^{\circ} \mathrm{C}$ in a humidified atmosphere of 5\% $\mathrm{CO}_{2}$. GSWW/2015 (GenBank accession number: KX767091.1, https://www.ncbi. nlm.nih.gov/nuccore/KX767091) was isolated by our laboratory and characterized as an HP-PRRSV strain. These PRRSV strains were propagated in Marc-145 cells, and viral titers were determined using $\mathrm{TCID}_{50}$ assay.

\section{RNA isolation, reverse transcription, and qPCR}

Total RNA was isolated using the RNeasy kit (Qiagen) according to the manufacturer's protocol. Equal amounts of total RNA were reverse transcribed using the PrimeScript $^{\mathrm{TM}}$ RT Master Mix (Takara). cDNA was then subjected to qPCR using TB Green ${ }^{\circledR}$ Premix Ex Taq ${ }^{\mathrm{TM}}$ II (Takara) on a QuantStudio 5 Real-Time PCR system (Applied Biosystems). Relative expression of lncRNAs and mRNAs was calculated using the comparative $\mathrm{Ct}$ method as previously described [34]. Primers for qPCR are listed in Additional file 8: Table S7.

\section{RNA-sequencing (sample preparation, library construction, and sequencing)}

A total of $1 \times 10^{7}$ PAMs were seeded in a $25 \mathrm{~cm}^{2}$ flask and cultured for $12 \mathrm{~h}$ before virus infection. Then PAMs were mock-infected or infected with GSWW and VR2332 at an MOI of 0.1 for $24 \mathrm{~h}$. Each group contains three biological repeats. Subsequently, cells were washed with PBS once and harvested. Total RNA was extracted using TRizol (Thermo Fisher) following the manufacturer's instruction. RNA concentration and purity were measured by Nanodrop 2000, and RNA integrity was assessed by the Bioanalyzer 2100 system (Agilent Technologies). In all, $3 \mu \mathrm{g}$ RNA per sample was used as input for the RNAsequencing library construction. The procedure was conducted as previously described [34]. Briefly, ribosomal RNA was removed, and residual RNAs were cleaned using ethanol precipitation. Nine sequencing libraries were generated using the NEBNext ${ }^{\circledR}$ Ultra $^{\mathrm{TM}}$ Directional RNA Library Prep Kit for Illumina ${ }^{\circledR}$ (NEB, USA). The quality of the libraries was checked on an Agilent Bioanalyser 2100 system. The libraries were sequenced on an Illumina Hiseq X TEN platform, and 150 bp paired-end reads were generated. The RNA-Seq and data collection were performed by Novogene Co. LTD, Beijing, China.

\section{Data analysis of RNA-sequencing}

Raw data of fastq format were firstly processed by inhouse Perl scripts of Novogen. Clean data were acquired by removing adapter sequence, reads containing poly- $\mathrm{N}$, and low-quality reads from raw data. Meanwhile, Q20, 
Q30, and GC content of the clean data were calculated. All the downstream analyses were based on clean data with high quality $(\mathrm{Q} 30>90 \%)$. The paired-end clean reads were aligned to the porcine reference genome (Sscrofa11.1) by TopHat v2.0.9. The mapped reads of each sample were assembled by Cufflinks (v2.1.1).

\section{LncRNA identification}

To identify lncRNAs, the assembled transcripts were first filtered by FPKM $>0.5$, coverage $>1$, and length $>200 \mathrm{nt}$ to remove background noise. Subsequently, four programs namely, CNCI (Coding-Non-Coding-Index), CPC (Coding Potential Calculator), Pfam, and phyloCSF (phylogenetic codon substitution frequency), were utilized to analyze the coding potential of filtered transcripts. CNCI profiles adjoining nucleotide triplets to discriminate protein-coding and non-coding sequences independent of known annotations. CPC (0.9-r2) mainly evaluates the extent and quality of the ORF in a transcript and searches the sequences with known protein sequence databases to distinguish the coding and non-coding transcripts [9]. Each transcript was translated in all three possible frames, and Pfam Scan was used to find the occurrence of any of the known protein family domains documented in the Pfam database [16]. Transcripts with a Pfam hit would be excluded in the following steps [2]. PhyloCSF scans evolutionary characteristics to alignments of conserved coding regions to clarify protein-coding and non-coding transcripts [13]. Transcripts predicted with coding potential by either of the four programs above were excluded.

\section{Differential gene expression analysis of RNA-Sequencing data}

The FPKM (fragments per kilobase per million reads) values of both lncRNAs and mRNAs in each sample were computed by Cuffdiff (v2.1.1) [24]. Gene expression levels were calculated by summing the FPKMs of transcripts in each gene group. Cuffdiff provides statistical routines for determining differential expression in gene expression data by a model based on the negative binomial distribution [24]. Transcripts with a $P$-value $<0.05$ were considered as differentially expressed genes.

\section{LncRNA target gene prediction}

Most of the porcine lncRNAs have not yet been functionally annotated. In this study, the prediction of their target genes is based on the functional annotations of their related cis and trans target mRNAs. Coding genes located within $100 \mathrm{~kb}$ upstream and downstream of lncRNAs in the genome were searched and assigned as lncRNA co-location genes (cis-activating lncRNAs). The expressed correlations between lncRNAs and coding genes were calculated with custom scripts. Coding genes and lncRNAs with Pearson correlation score $>0.95$ or $<-0.95$ were considered co-expressed (trans-activating lncRNAs). The functions of lncRNAs were predicted by functional enrichment analysis of their co-location and co-expressed coding genes.

GO and KEGG enrichment analysis of RNA-sequencing data Gene Ontology (GO) enrichment analysis of differently expressed mRNAs and lncRNA target genes were executed by the GOseq $\mathrm{R}$ package. GO terms with corrected $P$-value $<0.05$ were considered significantly enriched. KOBAS software was utilized to test the statistical enrichment of DE mRNAs and lncRNA target genes in KEGG pathways (Kyoto Encyclopedia of Genes and Genomes) [32].

\section{siRNA and cell transfection}

The siRNAs were purchased from GenePharma (China) and JTS BIO (China). The sequences of siRNAs are listed in Additional file 8: Table S7. siRNAs were transiently transfected with Lipofectamine 3000 (Thermo Fisher) according to the manufacturer's instructions. The amount of siRNA used for transfection was optimized to avoid cellular toxicity, and $50 \mathrm{nM}$ siRNA was used.

\section{Results \\ Identification of DE IncRNAs after PRRSV infection by RNA-sequencing}

To identify PRRSV-regulated lncRNAs in porcine primary alveolar macrophages, we performed RNASequencing of total RNA without rRNA extracted from PRRSV-infected PAMs. Specifically, PAMs isolated from three 4-week-old SPF pigs were infected with GSWW, VR2332 at an MOI of 0.1, or mock-infected were collected at 24 hpi. Samples were prepared from triplicates and nine sequencing libraries were constructed. Approximately 108 million (range from 91.6 to 119 million) $150 \mathrm{bp}$ paired-end clean reads were generated on average of each sample after removing adapter fragments and low-quality reads. Clean reads were aligned to Sus Scrofa (sscrofa 11.1) genome with TopHat. Transcripts were assembled using Cufflinks. The expression levels of transcripts were calculated by cuffdiff and shown as fragments per kilobase per million reads (FPKMs).

As shown in Fig. 1a, five steps were performed to identify novel lncRNAs. First, single-exon transcripts, which might be transcription noise, were filtered out. Second, transcripts longer than $200 \mathrm{bp}$ were kept according to the definition of lncRNA. Third, Cuffcompare was utilized to identify transcripts overlapped with exons of annotated mRNAs, which were assigned as annotated lncRNAs. We also explored 
a domestic-animal long non-coding RNA database (ALDB) to find annotated lncRNAs [10]. Then transcripts expressed at low levels $(\mathrm{FPKM}<0.5)$ were removed. Last, we used CNCI, CPC, Pfam-scan, and phyloCSF to predict the coding potential of transcripts. Transcripts predicted with coding potential by either of the four programs above were filtered out (Fig. 1b). After these steps, 1147 transcripts were predicted as novel lncRNAs.

Using cuffdiff, a total of 441 and 1509 differently expressed mRNAs (DE mRNAs) were identified between the GSWW and VR2332 groups, respectively (Additional file 2: Table S1). The numbers of differently expressed lncRNAs (DE lncRNAs) were less than mRNAs in both comparison groups, which was 101 in GSWW and 239 in the VR2332 infection group, respectively (Fig. 2a, b, and Additional file 3: Table S2). Figure 2c showed a heat map of 15 DE lncRNAs after PRRSV infection. The data demonstrated hierarchical clustering of $\log 2$ (FPKM) at each sample. We selected ten genes to validate their expression by RT-qPCR (Fig. 2d and Additional file 1: Figure S1). Five mRNAs and five lncRNAs were tested, the correlation between the RT-qPCR results and RNA-Seq data was measured by Pearson's correlation coefficient. A strong correlation between the two methods was observed for all the tested genes (Table 1).

\section{Prediction of IncRNA targets}

LncRNAs regulate the expression of their target mRNA through various mechanisms. It has been reported that lncRNAs could regulate the transcription of their neighboring protein-coding genes (cis-acting lncRNAs). We predicted the function of lncRNAs by analyzing protein-coding genes located within $100 \mathrm{~kb}$ upstream and downstream of lncRNAs in the genome (Additional file 6: Table S5). The mRNAs showed correlated expression patterns with differently expressed lncRNAs were more likely to be modulated by the lncRNAs (trans-acting lncRNAs). Therefore, we used Pearson correlation analysis to identify co-expressed lncRNAs and mRNAs in each comparison group. The lncRNA-mRNA pairs with Pearson correlation score $>0.95$ or $<-0.95$ were considered co-expression (Additional file 7: Table S6).

\section{GO enrichment and KEGG pathway analysis of DE mRNAs and predicted IncRNA targets}

To characterize PRRSV-infection-induced functional alterations of RNA expression profiles, we performed Gene Ontology enrichment analysis of DE mRNAs. Up-regulated genes were enriched in 33 and 16 biological processes upon GSWW or VR2332 infection, respectively. Most of them were related to virus infection and immune response such as immune effector process, response to virus, and defense response to virus. (Additional file 1: Figure S2 and S Additional file 4: Table S3). We also performed KEGG enrichment analysis of DE mRNAs to identify pathways involved in PRRSV infection. We found that up-regulated mRNAs of both groups were enriched in virus infectionrelated pathways such as Influenza A and Herpes simplex infection. DE mRNAs in the GSWW infection group were enriched in innate immune response pathways such as Toll-like receptor, RIG-I-like receptor, and NF-kB signaling pathway (Additional file 1: Figures S3-S5 and Additional file 5: Table S4).

Due to the lacking of functional annotation of porcine lncRNAs, KEGG analysis of co-localized and co-expressed mRNAs was utilized to predict IncRNA function. KEGG analyses of mRNAs co-localized with DE lncRNAs (cis-acting lncRNAs) in the GSWW infection group were enriched in immune response-related pathways such as Inflammatory bowel disease, Leishmainasis, Intestinal immune network for IgA production, and so on (Fig. 3a). There was no significantly enriched pathway of mRNAs co-localized with up-regulated lncRNAs in the VR2332 infection group. We only identified one pathway enriched in mRNAs colocalized with down-regulated lncRNAs in the VR2332 infection group, which was Inflammatory bowel disease (Fig. 3b). As for the functional prediction of trans-activating lncRNAs, we found that mRNAs co-expressed with upregulated lncRNAs were enriched in virus infection-related pathways such as Herpes simplex infection in both groups (Fig. 4 and Additional file 7: Table S6). We did not identify significantly enriched pathways of mRNAs co-expressed with down-regulated lncRNAs in both groups.

\section{Validation of IncRNAs function}

To further explore the function of these DE lncRNAs, we chose five up-regulated lncRNAs to study. Since it is technically challenging to force the expression of a foreign gene in PAMs, we reduced their transcription by transfecting

\footnotetext{
(See figure on next page.)

Fig. 1 a Five steps were performed to predict novel IncRNAs. The X-axis stood for each step, and Y-axis stood for the number of transcripts left after each step. First, single-exon transcripts were filtered out. Second, transcripts longer than 200 nt were kept. Third, using Cuffcompare, transcripts overlapped with exons of annotated mRNAs were assigned as annotated IncRNAs. Fourth, transcripts expressed at low levels (FPKM < 0.5) were removed. Fifth, four programs were applied to predict the coding potential of transcripts, namely CNCl, CPC, Pfam-scan, and phyloCSF. b The Venn diagram showed numbers of predicted non-coding transcripts by CNCI, CPC, Pfam-scan, and phyloCSF. A total of 1147 transcripts predicted as non-coding transcripts by all the algorithms were characterized as novel IncRNAs
} 
(a)

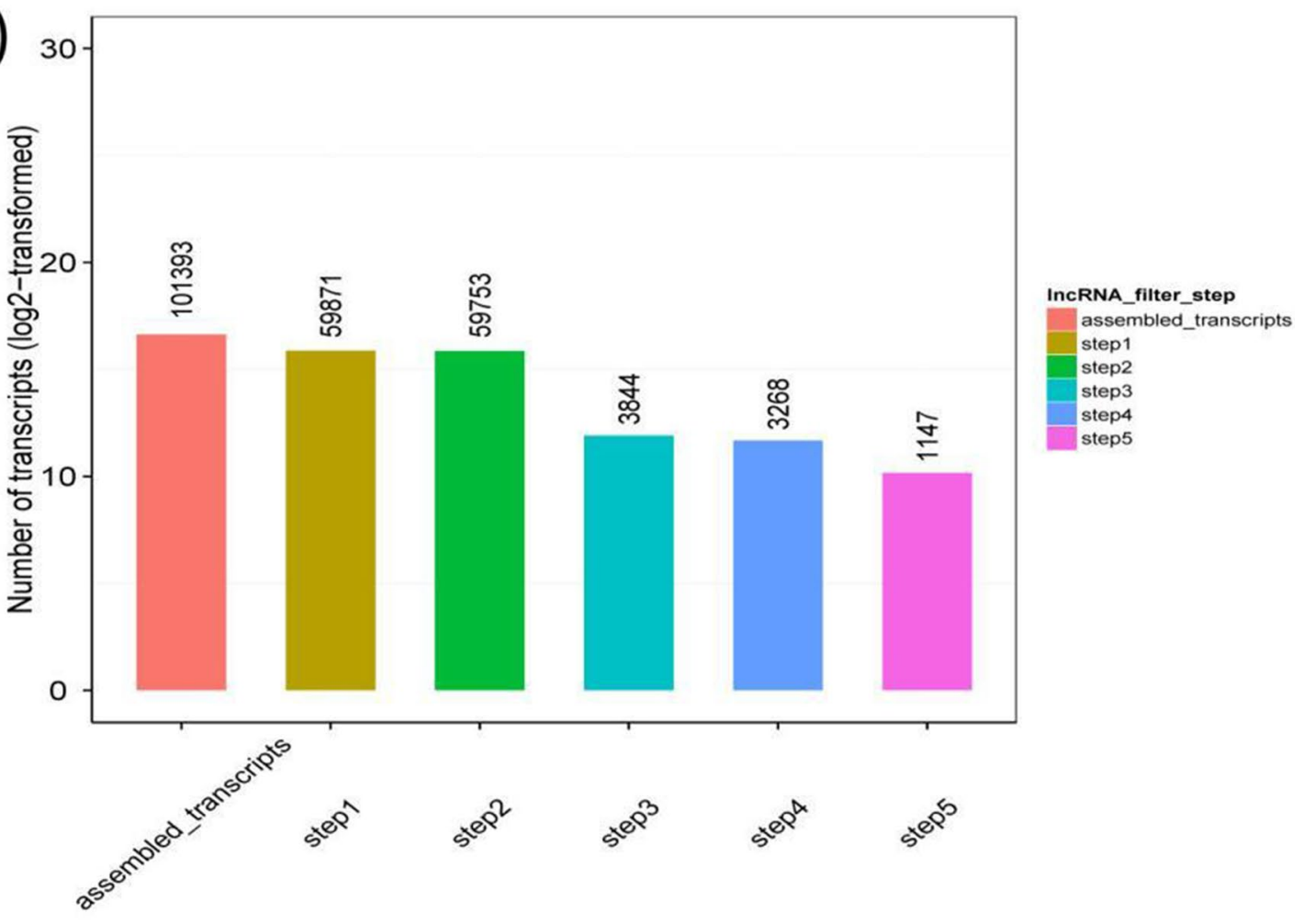

(b)

PFAM

phyloCSF

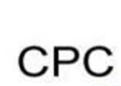

42

193

$\mathrm{CNCl}$

25

161

180

76

189

325

50

1147

222

109

156

194

54

Fig. 1 (See legend on previous page.) 


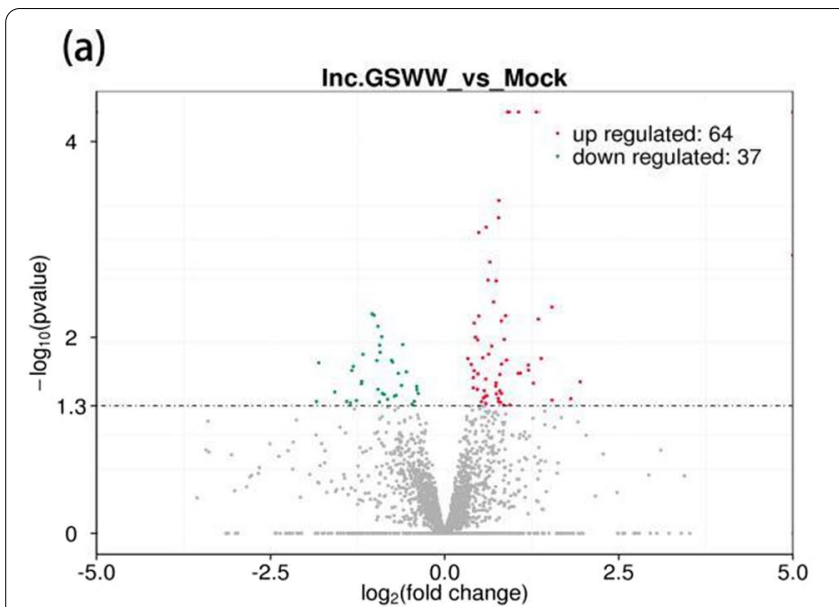

(c)

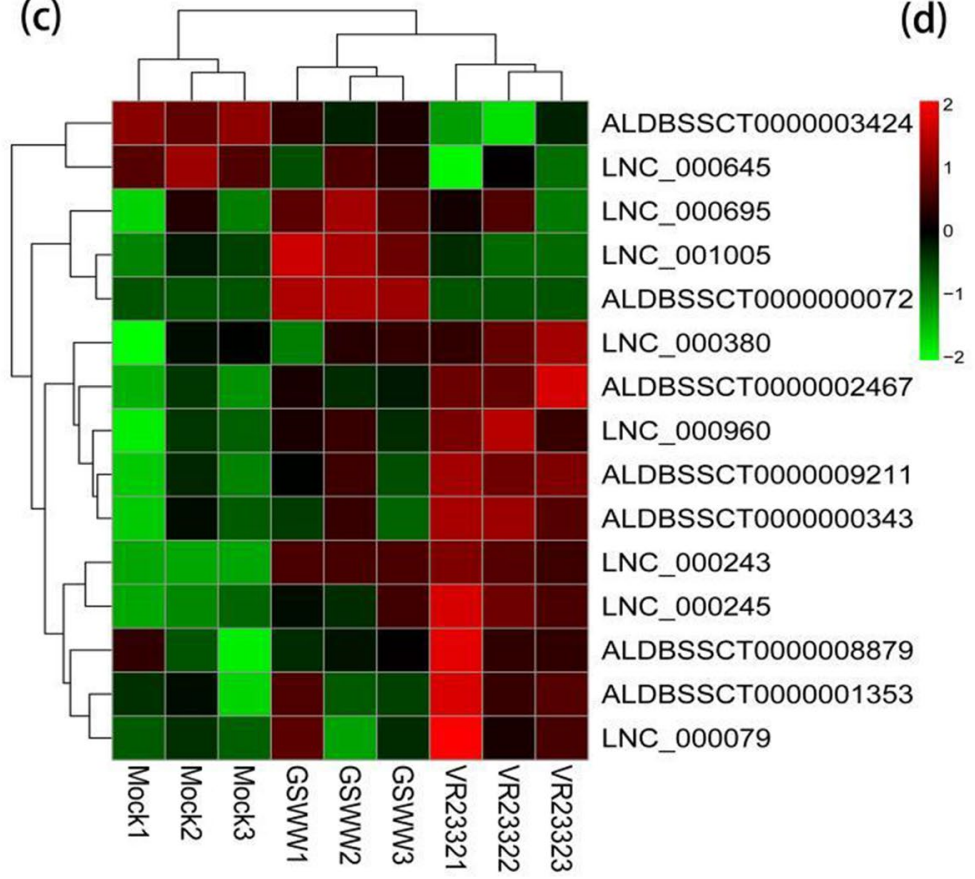

(b)

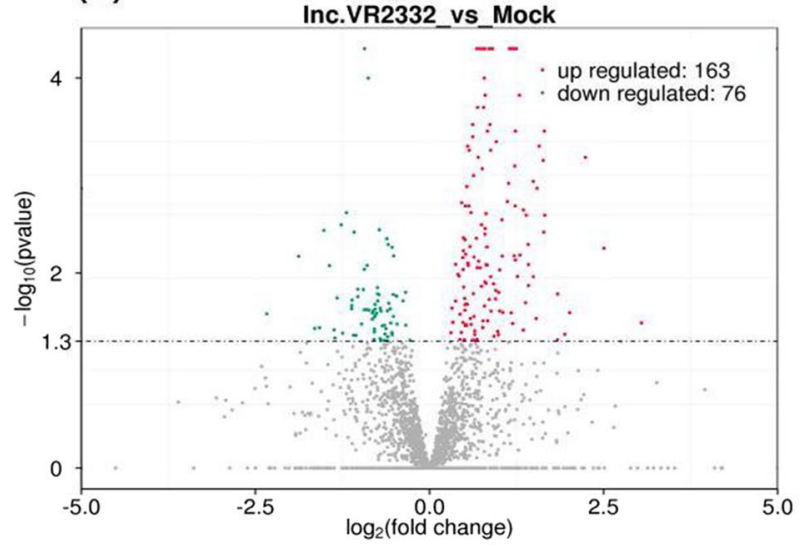

(d)

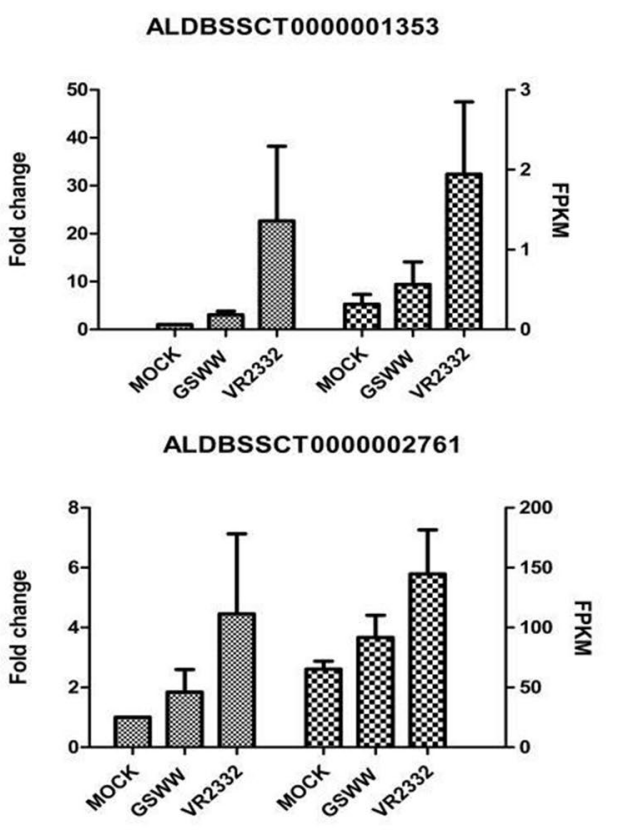

Fig. 2 Volcano plots show differentially expressed IncRNAs in a GSWW versus Mock comparison group and in b VR2332 vs. Mock comparison group. c Hierarchical heat map demonstrating transformed expression values (log2 FPKM) for 15 differentially expressed IncRNAs. Red shows up-regulation and green shows down-regulation. $\mathbf{d}$ RT-qPCR results of differentially expressed genes after PRRSV infection by GSWW and VR2332 at $24 \mathrm{hpi}$ in PAMs. Total RNA was extracted, and the first-strand cDNA was synthesized using reverse transcriptase kit. The bar represents the mean of three samples. Expression levels were normalized to GAPDH

siRNAs targeting these lncRNAs. After transfection, cells were infected with GSWW and VR2332, and virus copies numbers were analyzed by RT-PCR at $24 \mathrm{hpi}$ (Fig. 5a and b). Knockdown of LNC_000397 increased PRRSV RNA copy numbers, about twofold by GSWW infection and sixfold by VR2332 infecton (Fig. 5c and d). To validate the role of LNC_000397 in suppressing PRRSV replication, we also used $\mathrm{TCID}_{50}$ assay to determine virus titers. The results showed that knockdown of LNC_000397 increased virus titers on Marc-145 cells, about fivefold by GSWW infection and tenfold by VR2332 infection (Fig. 5e). LNC_000397 locates at chromosome 13, whose full length is $8540 \mathrm{nt}$. We cloned the full length of this lncRNA from PAMs and sent for sanger-sequencing. The sequence was identical to that got from RNA-Seq.

Then we inoculated PAMs with GSWW and VR2332 strains at an MOI of 0.1 and found that the LNC_000397 expression level was increased from $12 \mathrm{hpi}$ in a timedependent manner, the induction was most obvious at 36 hpi (about sixfold by VR2332 infection and 2.5 fold 
Table 1 Correlation analysis of RNA sequencing and RT-qPCR results

\begin{tabular}{|c|c|c|c|c|c|}
\hline Transcript name & & MOCK & GSWW & VR2332 & Correlation score \\
\hline \multirow[t]{2}{*}{ ALDBSSCT0000001353 } & FPKM & 0.317347 & 0.564727 & 1.943572 & 0.998 \\
\hline & RT-QPCR & 1 & 3.053109 & 22.66456 & \\
\hline \multirow[t]{2}{*}{ CD274 } & FPKM & 16.00107 & 25.69197 & 39.9606 & 0.998 \\
\hline & RT-QPCR & 1 & 1.771723 & 3.207748 & \\
\hline \multirow[t]{2}{*}{ TNFSF10 } & FPKM & 22.69663 & 44.223 & 97.9314 & 0.994 \\
\hline & RT-QPCR & 1 & 2.105087 & 7.100317 & \\
\hline \multirow[t]{2}{*}{ LNC_000397 } & FPKM & 0.517206 & 0.885413 & 1.229253 & 1.000 \\
\hline & RT-QPCR & 1 & 1.510961 & 2.025578 & \\
\hline \multirow[t]{2}{*}{ ALDBSSCT0000002761 } & FPKM & 65.2994 & 91.73363 & 144.6964 & 0.995 \\
\hline & RT-QPCR & 1 & 1.840139 & 4.459228 & \\
\hline \multirow[t]{2}{*}{ RNASEL } & FPKM & 4.922467 & 9.364923 & 21.53297 & 0.997 \\
\hline & RT-QPCR & 1 & 1.221049 & 1.654972 & \\
\hline \multirow[t]{2}{*}{ CD169 } & FPKM & 5.571897 & 14.4758 & 42.70057 & 0.997 \\
\hline & RT-QPCR & 1 & 1.715125 & 5.186836 & \\
\hline \multirow[t]{2}{*}{ PKR } & FPKM & 17.13503 & 42.66707 & 63.8304 & 1.000 \\
\hline & RT-QPCR & 1 & 2.667194 & 4.195808 & \\
\hline \multirow[t]{2}{*}{ LNC_000695 } & FPKM & 7.480513 & 15.6161 & 10.31643 & 0.999 \\
\hline & RT-QPCR & 1 & 1.562077 & 1.169119 & \\
\hline \multirow[t]{2}{*}{ LNC_000454 } & FPKM & 0.699077667 & 1.057696667 & 1.074059667 & 0.886 \\
\hline & RT-QPCR & 1 & 1.306818 & 1.174907 & \\
\hline
\end{tabular}

by GSWW infection) (Fig. 6a). Since VR2332 induced its expression more obviously, we infected PAMs with VR2332 at MOIs of 0.05, 0.1 and 0.5. The results showed that the expression of LNC_000397 was induced in a dose-dependent manner (Fig. 6b). To study whether induction of LNC_000397 depends on virus replication, we treated PAMs with UV-inactivated and heat-inactivated VR2332 and found that inactivated virus was not able to induce LNC_000397 expression (Fig. 6c). Poly(I: C) transfection also increased LNC_000397 expression in PAM (about ninefold) and PK-15 cells (about twofold) (Fig. 6d and e).

To study its functional mechanism, we utilized RNASeq to identify target genes of Lnc_000397. We found that top genes suppressed by LNC_000397 knockdown were interferon-stimulated genes that play essential roles in antiviral response, such as CXCL10 (C-X-C Motif Chemokine Ligand 10), IFIT2 (Interferon-Induced Protein With Tetratricopeptide Repeats 2), and RSAD2 (Radical S-Adenosyl Methionine Domain Containing 2). We validated the sequencing results by RT-qPCR. Our results showed that knockdown of LNC_000397 decreased more than $70 \%$ expression of these ISGs (Fig. 7a). Then we asked whether LNC_000397 expression is induced by interferon. We treated PAMs with IFN- $\alpha$ and TNF$\alpha$, the results showed that LNC_000397 expression was induced by IFN- $\alpha$ (about sevenfold in PAM and 2.5 foldin PK cells) but not TNF- $\alpha$ (Fig. 7b and c). These results indicated that LNC_000397 suppressed PRRSV replication by inducing the expression of ISGs, and it was also up-regulated by interferon treatment.

\section{Discussion}

PRRSV has caused tremendous economic losses to the world swine industry. The high mutation rate and immune suppression nature of the virus make it challenging for vaccine development. Further understanding of the interaction between PRRSV and the host immune system would be beneficial for the implementation of novel antiviral strategies. One of the main goals of this study was to explore the role of host lncRNAs in regulating PRRSV-induced immune response. In this research, we characterized a total of 293 differently expressed lncRNAs after infection with two PRRSV strains. Inconsistent with our and other groups' reports, predicted targets of DE lncRNAs were enriched in immune-response related pathways such as NF-kB and RIG-I signaling [7, 34].

Importantly, we identified a lncRNA, LNC_000397, suppressed PRRSV replication in PAMs. The expression of lnc_000397 was up-regulated in PRRSV GSWW and VR2332 infected cells. It also could be induced by poly (I: C), a synthetic dsRNA analog, in PAM and PK15 cells. Using RNA-seq, we found that knocking down of this lncRNA down-regulated the expression of ISGs such as CXCL10, MX1, ISG15, and RSAD2. Furthermore, we demonstrated that LNC_000397 was 


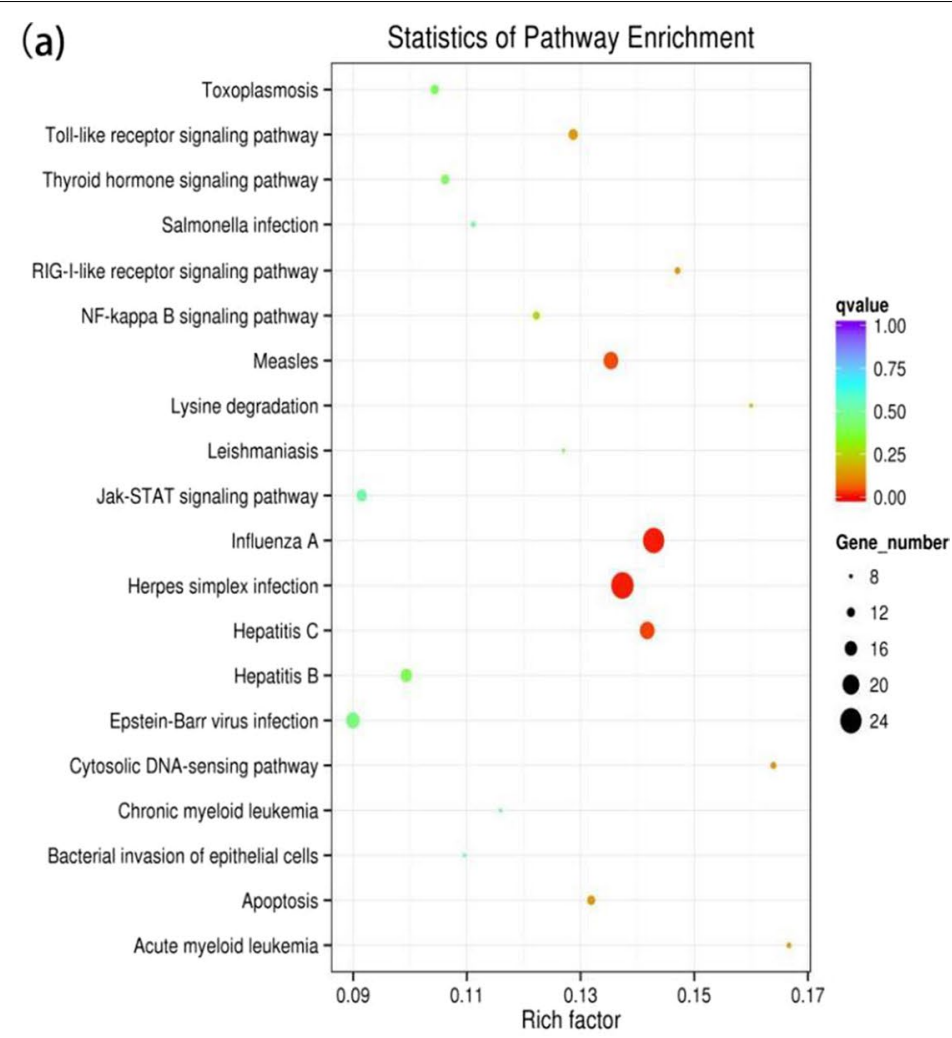

\section{(b)}

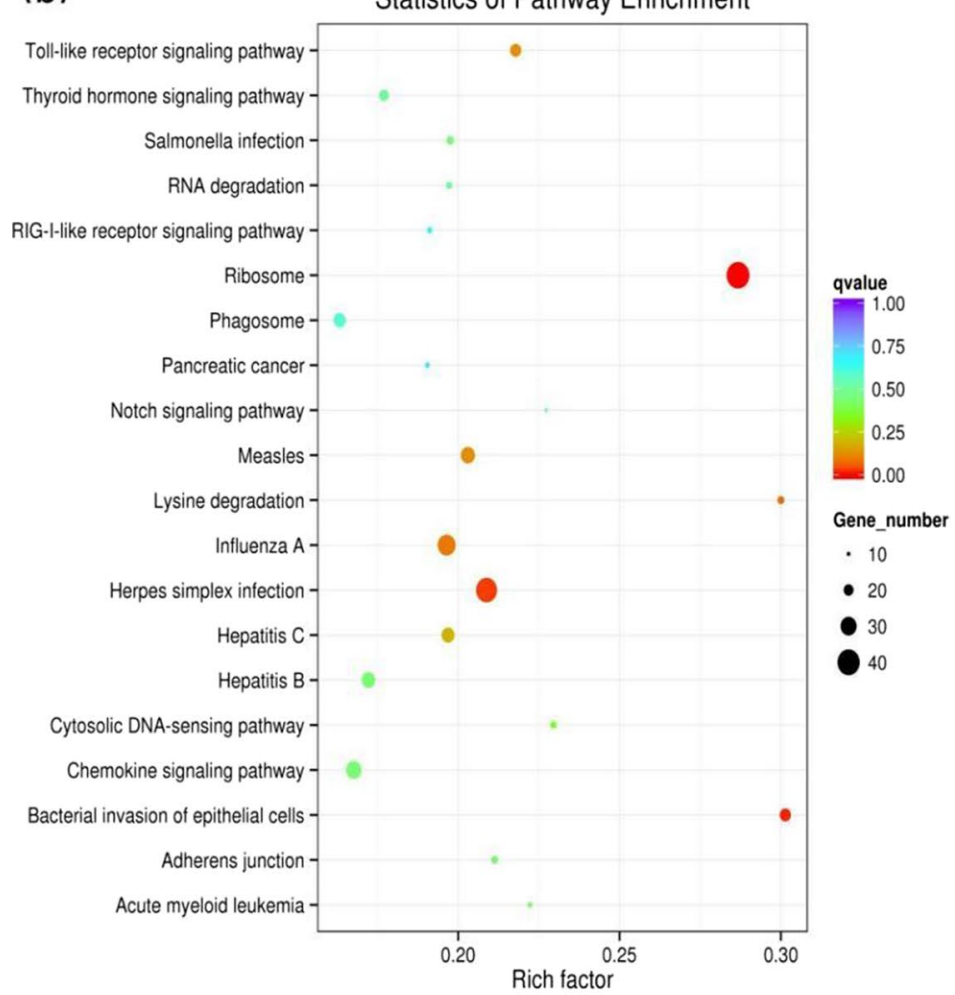

Fig. 3 Scatter plots of KEGG pathway enrichment statistics. Top 20 statistics of pathways, enrichment in the KEGG database for neighboring mRNAs of differentially expressed IncRNAs in a the GSWW infection group and the $\mathbf{b}$ VR2332 infection group 


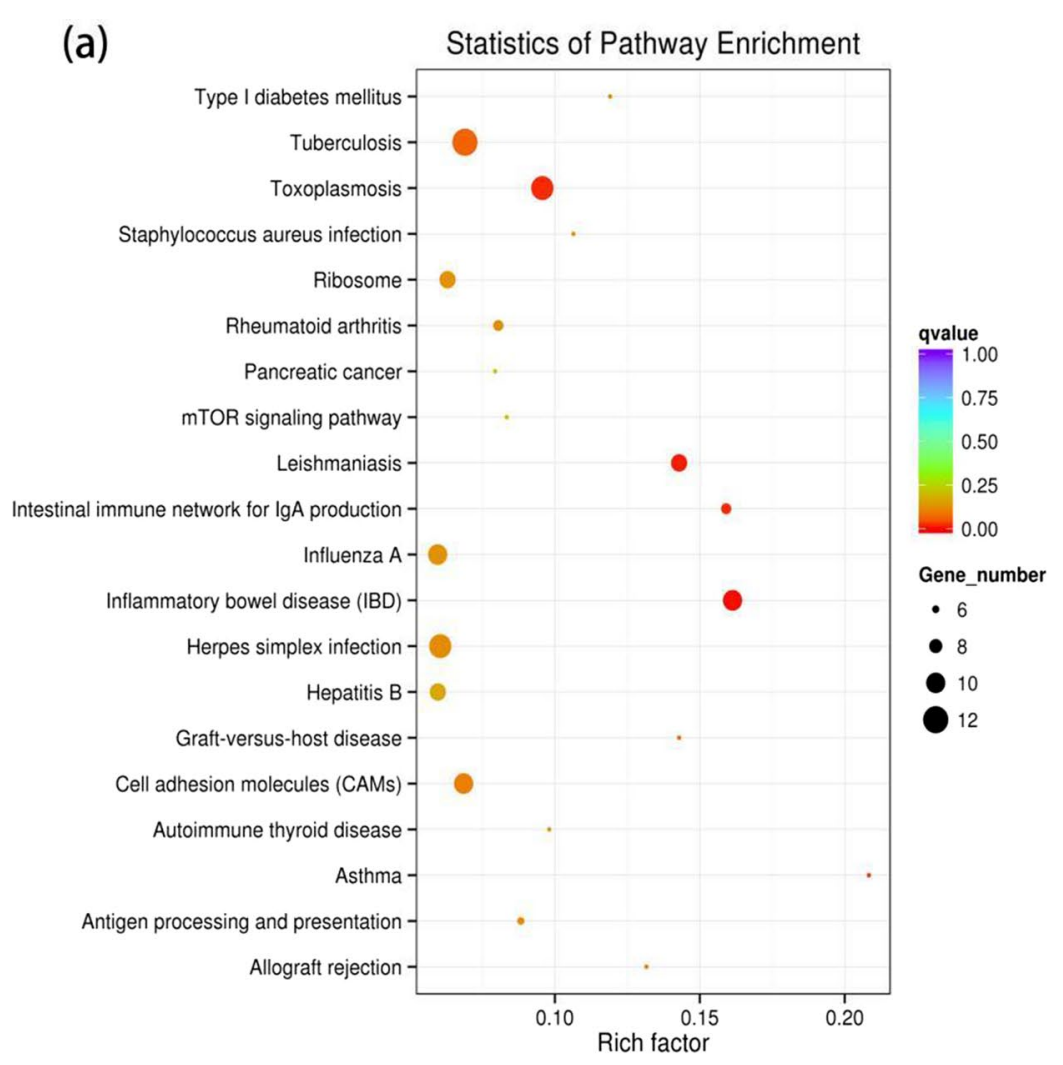

(b)

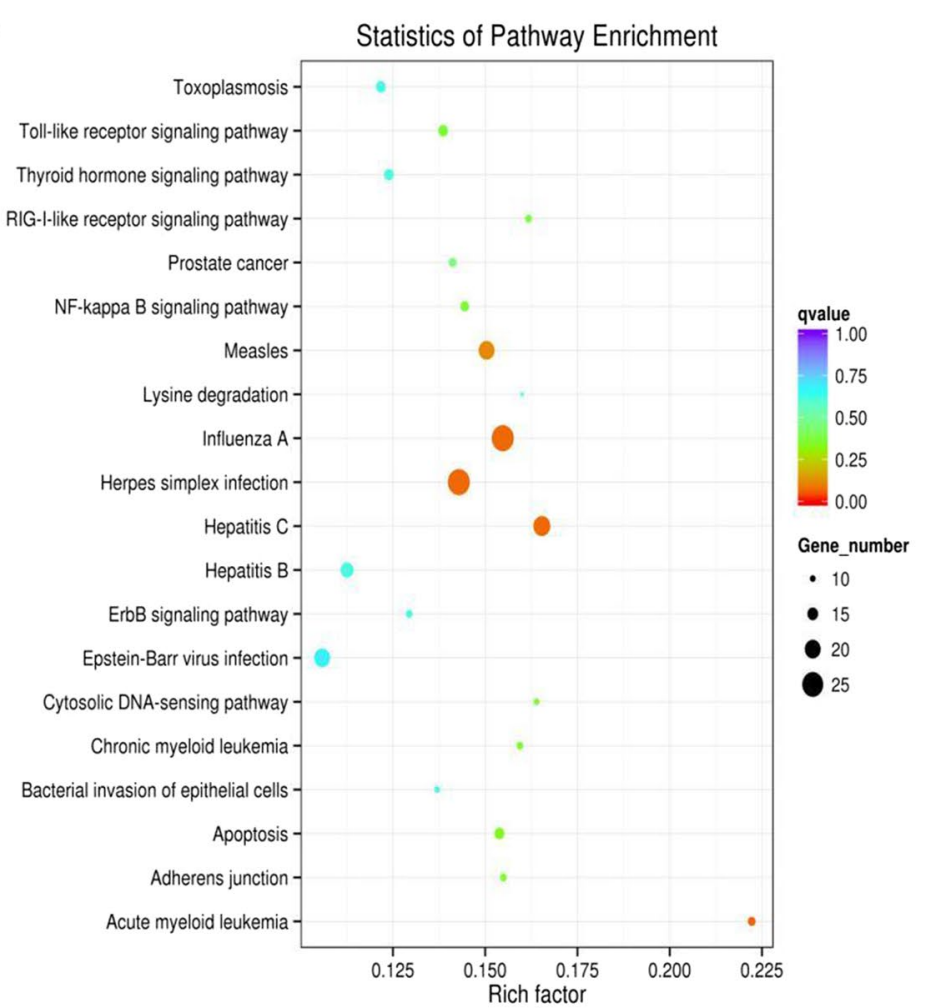

Fig. 4 Scatter plots of KEGG pathway enrichment statistics. Top 20 statistics of pathways, enrichment in the KEGG database for co-expressed mRNAs of differentially expressed IncRNAs in $\mathbf{a}$ the GSWW infection group and $\mathbf{b}$ the VR2332 infection group 


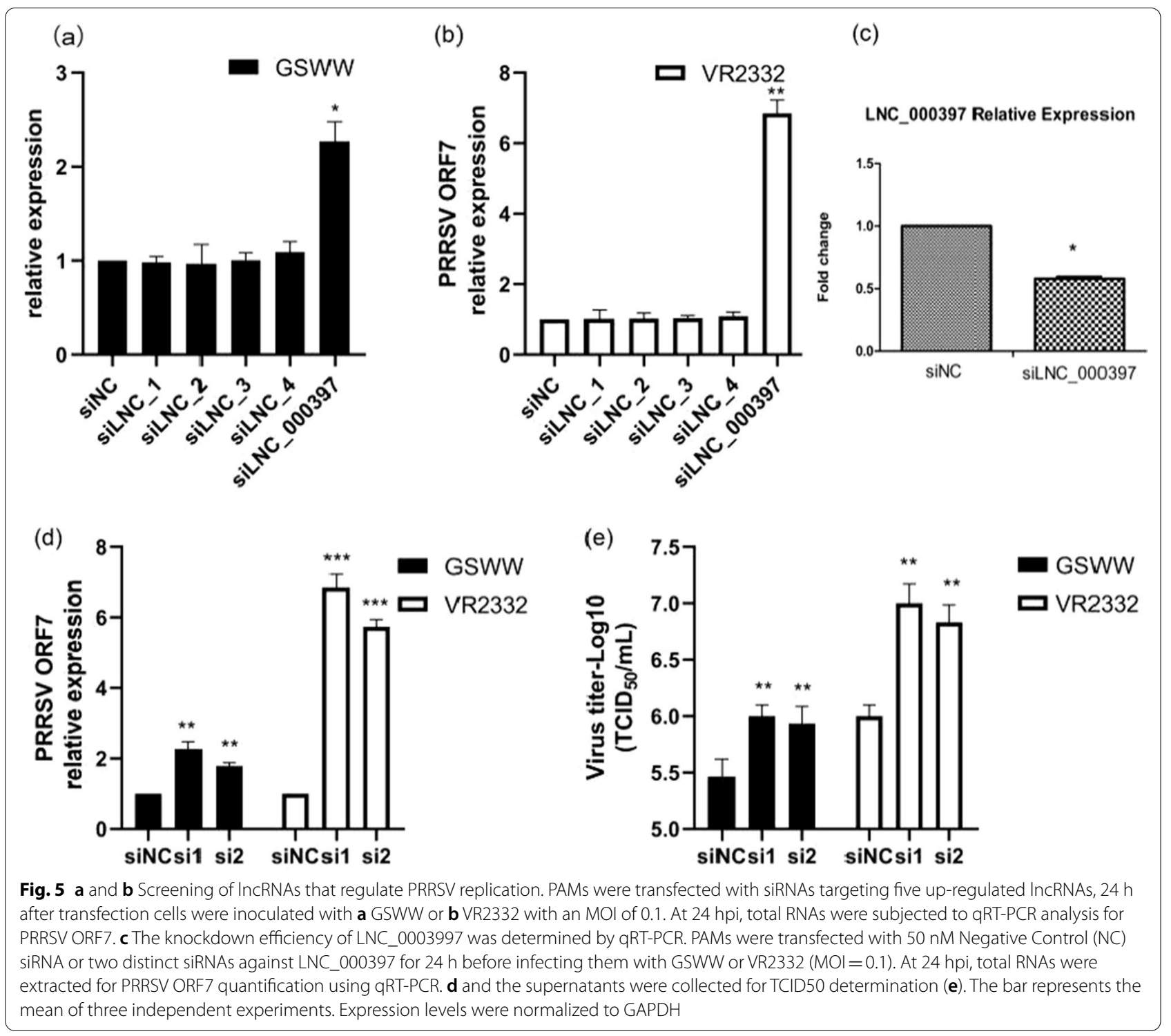

induced by type I-IFN treatment. Because of the low transfection efficiency of primary macrophage, we could not detect the effect of LNC_000397 over-expression on PRRSV replication. Our results suggested that LNC_000397 is an IFN-dependent antiviral lncRNA.

Interferon signaling is one of the most critical parts of innate immunity to defense against virus infection. To date, the vast majority of ISGs involved in the antiviral immune response are proteins, such as $\mathrm{Mx}$, IFIT protein family, and OAS protein family. In recent years, the roles of interferon-dependent lncRNAs have emerged. An IFN- $\beta$ induced lncRNA, lnc-ISG20, inhibits IAV replication by enhancing ISG20 expression [3]. Lnc-MxA is an interferon-stimulated gene (ISG) functions as a negative regulator of the antiviral immune response [11]. These reports indicated that lncRNAs represent another set of ISGs that exert important roles in the antiviral immune response. Our studies showed that LNC_000397 was stimulated by PRRSV infection and negatively regulated virus replication as a novel ISG.

\section{Conclusions}

Our study has shown that LNC_000397 was up-regulated by PRRSV infection in PAMs and impaired PRRSV replication by up-regulating ISGs expression. This research is the first report revealing the role of a lncRNA in regulating PRRSV replication, which might provide new insights for the development of novel antiviral strategies. 

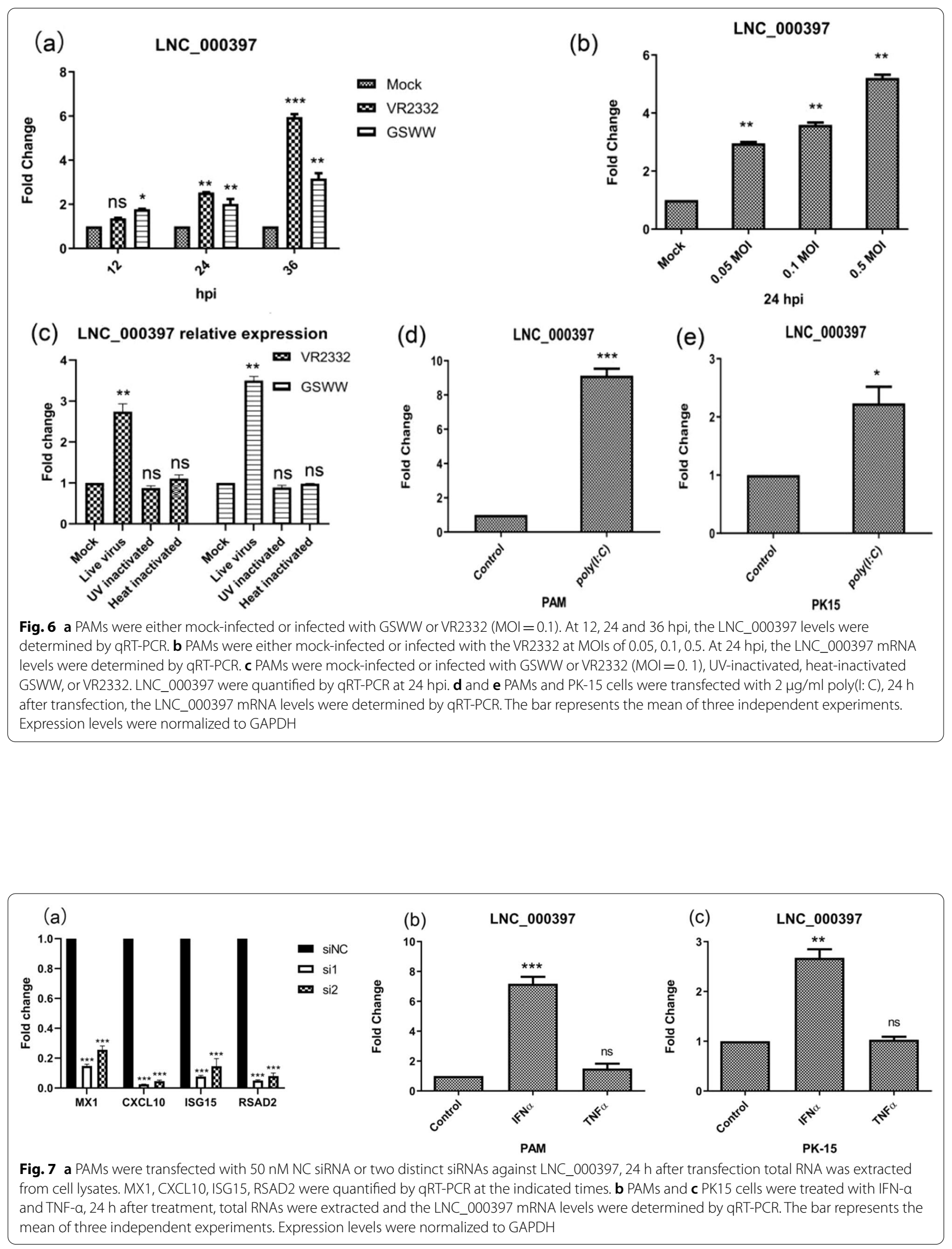


\section{Abbreviations}

PRRSV: Porcine reproductive and respiratory syndrome virus; IncRNAs: Long non-coding RNAs; ISGs: Interferon-stimulated genes; siRNAs: Short interfering RNAs; MX1: MX Dynamin Like GTPase 1; CXCL10: C-X-C Motif Chemokine Ligand 10; RSAD2: Radical S-Adenosyl Methionine Domain Containing 2.

\section{Supplementary Information}

The online version contains supplementary material available at https://doi. org/10.1186/s12985-022-01761-x.

Additional file 1: Figure S1 RT-qPCR results of eight differentially expressed genes after PRRSV infection by GSWW and VR2332 at 24 hpi. Total RNA was extracted by Trizol, and the first strand cDNA was synthesized using reverse transcriptase kit. Bar represents the mean of three samples. Expression levels were normalized to GAPDH. Figure \$2 shows GO enrichment of up-regulated mRNAs in GSWW (a) and VR2332 (b) infected groups. Figure S3 shows Scatter plots of KEGG pathway enrichment statistics. Top 20 statistics of pathways, enrichment in the KEGG database for up-regulated mRNAs in GSWW infection group. Figure S4 shows Scatter plots of KEGG pathway enrichment statistics. Top 20 statistics of pathways, enrichment in the KEGG database for up-regulated mRNAs in VR2332 infection group. Figure S5 shows Scatter plots of KEGG pathway enrichment statistics. Top 20 statistics of pathways, enrichment in the KEGG database for down-regulated mRNAs in VR2332 infection group.

Additional file 2: Table S1 Lists differently expressed mRNAs upon GSWW and VR2332 infection compared to Mock infection.

Additional file 3: Table S2 Lists differently expressed IncRNAs upon GSWW and VR2332 infection compared to Mock infection.

Additional file 4: Table S3 Lists GO enrichment of up-regulated and down-regulated mRNAs in GSWW and VR2332 infected groups.

Additional file 5: Table S4 Lists KEGG pathway enrichment of up-regulated and down-regulated mRNAs in GSWW and VR2332 infected groups.

Additional file 6: Table S5 Lists KEGG pathway enrichment of mRNAs co-localized with differently expressed IncRNAs in GSWW and VR2332 infected groups.

Additional file 7: Table S6 Lists KEGG pathway enrichment of mRNAs co-expressed with differently expressed IncRNAs in GSWW and VR2332 infected groups.

Additional file 8: Table S7 Lists Real-time primers and siRNAs used in this study.

\section{Acknowledgements}

We thank Novogen for providing technical assistance in RNA sequencing and bioinformatics analysis. We also thank BMKCloud for providing bioinformatics analysis.

\section{Authors' contributions}

$J Z$, ZJL, and ZXL conceived and designed the study project; JZ, LPG, JW, PS, and $\mathrm{DL}$ performed the experiments, YMC, YFF, PHL, XWB, and $\mathrm{KL}$ analyzed the data. JZ prepared the manuscript. XQM, HFB, YLC, Jie Z, ZJL, and ZXL contributed to results discussion and data interpretation. All authors read and approved the final manuscript.

\section{Funding}

This work is supported by the Natural Science Foundation of Gansu Province (20JR10RA022) and the Key R\&D Program of Gansu Province (20YF3NA005).

\section{Availability of data and materials}

Accession codes for GSWW_2015_full_GENOME sequence: GenBank No. KX767091. Accession codes for RNA Sequencing: BioProject ID PRJNA716767. To whom requests for materials should be addressed (email: luzengjun@caas. $\mathrm{cn})$.

\section{Declarations}

\section{Ethics approval and consent to participate}

This study was conducted in strict accordance with the recommendations in the Guide for the Institutional Animal Care and Use Commission (IACUC). Animal experiments were approved by the Animal Ethics Committee of Lanzhou Veterinary Research Institute (LVRI), Chinese Academy of Agricultural Sciences.

\section{Consent for publication}

Not applicable.

\section{Competing interests}

The authors declare that they have no competing interests.

Received: 2 August 2021 Accepted: 21 February 2022

Published online: 05 March 2022

\section{References}

1. Badaoui B, Rutigliano T, Anselmo A, Vanhee M, Nauwynck H, Giuffra E, Botti S. RNA-sequence analysis of primary alveolar macrophages after in vitro infection with porcine reproductive and respiratory syndrome virus strains of differing virulence. PLOS ONE. 2014;9:91918.

2. Bateman A, Birney E, Cerruti L, Durbin R, Etwiller L, Eddy SR, Griffiths-Jones S, Howe KL, Marshall M, Sonnhammer ELL. The Pfam protein families database. Nucleic Acids Res. 2002;30:276-80.

3. Chai WJ, Li J, Shangguan QL, Liu Q, Li XD, Qi DD, Tong XM, Liu WJ, Ye X. Lnc-ISG20 inhibits influenza a virus replication by enhancing ISG20 expression. J Virol. 2018;92:0053918.

4. Chen J, Shi XB, Zhang XZ, Wang AP, Wang L, Yang YY, Deng RG, Zhang GP. MicroRNA 373 facilitates the replication of porcine reproductive and respiratory syndrome virus by its negative regulation of type I interferon induction. J Virol. 2017;91:01311.

5. Dokland T. The structural biology of prrsv. Virus Res. 2010;154(1-2):86-97.

6. Guttman M, Amit I, Garber M, French C, Lin MF, Feldser D, Huarte M, Zuk O, Carey BW, Cassady JP, Cabili MN, Jaenisch R, Mikkelsen TS, Jacks T, Hacohen N, Bernstein BE, Kellis M, Regev A, Rinn JL, Lander ES. Chromatin signature reveals over a thousand highly conserved large non-coding RNAs in mammals. Nature. 2009;458:223-7.

7. Gao J, Pan Y, Xu Y, Zhang W, Wang Y. Unveiling the long non-coding RNA profile of porcine reproductive and respiratory syndrome virus-infected porcine alveolar macrophages. BMC Genomics. 2021;22:177.

8. Kim O, Sun Y, Lai FW, Song C, Yoo D. Modulation of type I interferon induction by porcine reproductive and respiratory syndrome virus and degradation of CREB-binding protein by non-structural protein 1 in MARC-145 and HeLa cells. Virology. 2010;402:315-26.

9. Kong L, Zhang Y, Ye ZQ, Liu XQ, Zhao SQ, Wei L, Gao G. CPC: assess the protein-coding potential of transcripts using sequence features and support vector machine. Nucleic Acids Res. 2007;35:W345-9.

10. Li AM, Zhang JY, Zhou ZY, Wang L, Liu YJ, Liu YJ. ALDB: a domestic-animal long noncoding RNA database. PLOS ONE. 2015;10:0124003.

11. Li X, Guo G, Lu M, Chai W, Ye X. Lnc-MxA inhibits IFN- $\beta$ transcription via forming RNA-DNA triplexes at its promoter. J Virol. 2019;93:00786.

12. Li Y, Wang $X$, Bo K, Wang $X$, Tang B, Yang B, Jiang W, Jiang P. Emergence of a highly pathogenic porcine reproductive and respiratory syndrome virus in the Mid-Eastern region of China. Vet J. 2007;174:577-84.

13. Lin MF, Jungreis I, Kellis M. PhyloCSF: a comparative genomics method to distinguish protein coding and non-coding regions. Bioinformatics. 2011;27:1275-82.

14. Luo XG, Chen XX, Qiao SL, Li R, Xie S, Zhou XY, Deng RG, Zhou EM, Zhang GP. Porcine reproductive and respiratory syndrome virus enhances self-replication via AP-1-dependent induction of SOCS1. J Immunol. 2020;204:394-407.

15. Mercer TR, Dinger ME, Mattick JS. Long non-coding RNAs: insights into functions. Nat Rev Genet. 2009;10:155-9.

16. Mistry J, Chuguransky S, Williams L, Qureshi M, Salazar GA, Sonnhammer ELL, Tosatto SCE, Paladin L, Raj S, Richardson LJ, Finn RD, Bateman A. Pfam: The protein families database in 2021. Nucleic Acids Res. 2021;49:D412-9. 
17. Nishitsuji H, Ujino S, Yoshio S, Sugiyama M, Mizokami M, Kanto T, Shimotohno K. Long noncoding RNA \#32 contributes to antiviral responses by controlling interferon-stimulated gene expression. Proc Natl Acad Sci USA. 2016;113:10388-93.

18. Ouyang J, Zhu X, Chen Y, Wei H, Chen Q, Chi X, Qi B, Zhang L, Zhao Y, Gao GF, Wang G, Chen JL. NRAV, a long noncoding RNA, modulates antiviral responses through suppression of interferon-stimulated gene transcription. Cell Host Microbe. 2014;16:616-26.

19. Sagong $M$, Lee $C$. Porcine reproductive and respiratory syndrome virus nucleocapsid protein modulates interferon-beta production by inhibiting IRF3 activation in immortalized porcine alveolar macrophages. Arch Virol. 2011;156:2187-95.

20. Satpathy AT, Chang HY. Long noncoding RNA in hematopoiesis and immunity. Immunity. 2015;42:792-804.

21. Snijder EJ, Kikkert M, Fang Y. Arterivirus molecular biology and pathogenesis. J Gen Virol. 2013;94:2141-63.

22. Sun Y, Han MY, Kim C, Calvert JG, Yoo D. Interplay between interferonmediated innate immunity and porcine reproductive and respiratory syndrome virus. Viruses-Basel. 2012;4:424-46.

23. Sun Z, Chen Z, Lawson SR, Fang Y. The cysteine protease domain of porcine reproductive and respiratory syndrome virus nonstructural protein 2 possesses deubiquitinating and interferon antagonism functions. J Virol. 2010;84:7832-46

24. Trapnell C, Williams BA, Pertea G, Mortazavi A, Kwan G, van Baren MJ, Salzberg SL, Wold BJ, Pachter L. Transcript assembly and quantification by RNA-Seq reveals unannotated transcripts and isoform switching during cell differentiation. Nat Biotechnol. 2010;28:511-U174.

25. Wang J, Zhang YX, Li QJ, Zhao JY, Yi DR, Ding JW, Zhao F, Hu SQ, Zhou JM, Deng T, Li XY, Guo F, Liang C, Cen S. Influenza virus exploits an interferonindependent IncRNA to preserve viral RNA synthesis through stabilizing viral RNA polymerase PB1. Cell Rep. 2019;27:3295.

26. Wang P, Xu JF, Wang YJ, Cao XT. An interferon-independent IncRNA promotes viral replication by modulating cellular metabolism. Science. 2017;358:1051-5

27. Wang $Y$, Jing $N$, Willard $B$, Xin W, Stark GR. Negative regulation of type $i$ ifn signaling by phosphorylation of stat2 on t387. EMBO J. 2017;36:202-12.

28. Wensvoort G, Terpstra C, Pol JM, ter Laak EA, Bloemraad M, de Kluyver EP, Kragten C, van Buiten L, den Besten A, Wagenaar F, et al. Mystery swine disease in the Netherlands: the isolation of Lelystad virus. Vet Q. 1991;13:121-30.

29. Wilkinson JM, Bao H, Ladinig A, Hong LJ, Stothard P, Lunney JK, Plastow GS, Harding JCS. Genome-wide analysis of the transcriptional response to porcine reproductive and respiratory syndrome virus infection at the maternal/fetal interface and in the fetus. BMC Genomics. 2016;17:1-7.

30. Wu JJ, Peng XW, Qiao M, Zhao HZ, Li MB, Liu GS, Mei SQ. Genome-wide analysis of long noncoding RNA and mRNA profiles in PRRSV-infected porcine alveolar macrophages. Genomics. 2020;112:1879-88.

31. Xiao SQ, Wang X, Ni HB, Li N, Zhang AK, Liu HL, Pu FX, Xu LL, Gao JM, Zhao Q, Mu Y, Wang CB, Sun YN, Du TF, Xu XG, Zhang GP, Hiscox JA, Goodfellow IG, Zhou EM. MicroRNA miR-24-3p promotes porcine reproductive and respiratory syndrome virus replication through suppression of heme oxygenase-1 expression. J Virol. 2015;89:4494-503.

32. Xie C, Mao XZ, Huang JJ, Ding Y, Wu JM, Dong S, Kong L, Gao G, Li CY, Wei LP. KOBAS 2.0: a web server for annotation and identification of enriched pathways and diseases. Nucleic Acids Res. 2011;39:W316-22.

33. Yang $L$, He J, Wang R, Zhang X, Lin S, Ma Z, Zhang Y. Nonstructural protein 11 of porcine reproductive and respiratory syndrome virus induces STAT2 degradation to inhibit interferon signaling. J Virol. 2019;93:01352.

34. Zhang J, Sun P, Gan L, Bai W, Wang Z, Li D, Cao Y, Fu Y, Li P, Bai X, Ma X, Bao H, Chen Y, Liu Z, Lu Z. Genome-wide analysis of long noncoding RNA profiling in PRRSV-infected PAM cells by RNA sequencing. Sci Rep. 2017;7:4952.

35. Zhen YR, Wang FQ, Liang W, Liu JJ, Gao GL, Wang Y, Xu XW, Su QJ, Zhang QD, Liu B. Identification of differentially expressed non-coding RNA in porcine alveolar macrophages from Tongcheng and large white pigs responded to PRRSV. Sci Rep-Uk. 2018;8:1-11.

36. Zhou L, Wang Z, Ding Y, Ge X, Guo X, Yang H. NADC30-like strain of porcine reproductive and respiratory syndrome virus, China. Emerg Infect Dis. 2015;21:2256-7.

\section{Publisher's Note}

Springer Nature remains neutral with regard to jurisdictional claims in published maps and institutional affiliations.
Ready to submit your research? Choose BMC and benefit from:

- fast, convenient online submission

- thorough peer review by experienced researchers in your field

- rapid publication on acceptance

- support for research data, including large and complex data types

- gold Open Access which fosters wider collaboration and increased citations

- maximum visibility for your research: over $100 \mathrm{M}$ website views per year

At BMC, research is always in progress.

Learn more biomedcentral.com/submissions 